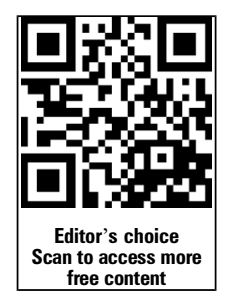
Research Core and the UCLA Stroke Center, Los Angeles, California, USA

2UCLA Stroke Center, Los Angeles, California, USA ${ }^{3}$ Department of Neurology, Emory University School of Medicine, Atlanta, Georgia, USA

${ }^{4}$ UPMC Stroke Center, Pittsburgh, Pennsylvania, USA ${ }^{5}$ OHSU Stroke Center, Portland, Oregon, USA

\section{Correspondence to} Dr David S Liebeskind, Neurovascular Imaging Research Core, UCLA Department of Neurology, Neuroscience Research Building, 635 Charles E Young Drive South, Suite 225, Los Angeles, CA 90095-7334, USA davidliebeskind@yahoo.com

Received 19 March 2015 Revised 20 April 2015 Accepted 23 April 2015 Published Online First 11 May 2015

\section{(a) CrossMark}

To cite: Liebeskind DS Jahan R, Nogueira RG, et al. J Neurolntervent Surg 2016:8:553-558.

\title{
Early arrival at the emergency department is associated with better collaterals, smaller established infarcts and better clinical outcomes with endovascular stroke therapy: SWIFT study
}

\author{
David S Liebeskind, ${ }^{1}$ Reza Jahan, ${ }^{2}$ Raul G Nogueira, ${ }^{3}$ Tudor G Jovin, ${ }^{4}$ \\ Helmi L Lutsep, ${ }^{5}$ Jeffrey L Saver, ${ }^{2}$ for the SWIFT Investigators
}

\begin{abstract}
Background and purpose Increasing time from symptom onset to emergency department arrival may incur greater ischemic injury and decreased likelihood of good outcomes after acute stroke therapy. The impact of time may be assessed bythe extent of acute CT changes, status of collateral vessels, and clinical outcomes.

Methods The SOLITAIRE FR With the Intention For Thrombectomy (SWIFT) trial comparing two neurothrombectomy treatments was analyzed by time, Alberta Stroke Program Early CT Scores (ASPECTS), angiographic collaterals, and 90-day modified Rankin Scale outcomes. We determined the interaction of time with ASPECTS, collateral grade, reperfusion, and clinical outcomes, with established determinants of angiographic and clinical outcomes as covariates.
\end{abstract}

Results 137 patients (52\% female) of mean age 67 \pm 12 years and median pretreatment NIH Stroke Scale score 18 (range 8-28) were enrolled. Median onset to door (OTD) time was 180 min (IQR 95-250).

Presentation within $3 \mathrm{~h}$ of last known well was associated with absence of any prestroke disability and presence of atrial fibrillation but was unrelated to age, sex, other vascular risk factors, deficit severity, glucose level, or blood pressure. Worse collaterals were noted with longer OTD intervals: collateral grade $0-1(n=32)$ : mean $232 \pm 84$ min; grade $2(n=48): 164 \pm 99$ min; grade $3(n=35): 155 \pm 104$ min; grade $4(n=4): 54 \pm 16$ min $(p<0.001)$. Later presentation was associated with more extensive early infarct imaging changes (median ASPECTS 8 (IQR 7-9) $>3 \mathrm{~h}$ vs 9 (IQR $8-10)<3 \mathrm{~h}$, $p=0.015$ ). Multivariable analyses identified time $>3 \mathrm{~h}$ as the only predictor of extensive infarct on imaging (ASPECTS $\leq 7$ ), $p=0.003$. Earlier presentation was strongly associated with better 90-day modified Rankin Scale outcomes $(p<0.001)$.

Conclusions Time was a critical factor in successful clinical outcomes for neurothrombectomy in the SWIFT trial. Shorter times to presentation were associated with better collaterals, smaller established infarcts, and better clinical outcome after revascularization.

\section{INTRODUCTION}

Emergency medicine plays a pivotal role in the initial triage and management of acute stroke. Diminishing long-term disability after acute ischemic stroke is contingent on timely diagnosis and decision-making regarding potential therapies, including revascularization. Reperfusion therapies may improve clinical outcomes after acute ischemic stroke, yet patient-specific factors including the baseline pathophysiology of individual patients may determine neurological sequelae. For instance, the status of collateral circulation in the brain may limit ischemic injury and greatly influence the response to treatments such as revascularization. ${ }^{1}$ The duration of ischemia or time from symptom onset to evaluation in the emergency department may also affect the severity or extent of infarction. Recanalization or opening an artery with endovascular stroke therapy may have different consequences due to such factors, including baseline pathophysiology and triage strategies.

Endovascular therapies for stroke have been studied for many years, culminating in the recent success of the MR CLEAN trial after many trials that attempted to refine revascularization, then failed to demonstrate better clinical outcomes. ${ }^{2-5}$ Previous trials used older devices and largely neglected baseline pathophysiology, whereas post hoc analyses of collateral status revealed important clues. ${ }^{1}$ Collateral grade and the extent of ischemic changes on CT could provide influential data in acute decision-making for stroke patients, including options such as endovascular therapy. The relationship between the time interval to emergency department arrival, subsequent measures of collaterals or CT changes, and ultimate outcomes after endovascular therapy are therefore important to discern. In fact, such variables may be critically important to achieve good outcomes with endovascular therapy when translated into routine clinical practice.

The SOLITAIRE FR With the Intention For Thrombectomy (SWIFT) study was a landmark trial that demonstrated the superiority of stent-retriever device technology over the predicate coil retrieval device for recanalization of occluded arteries in the setting of acute ischemic stroke. ${ }^{6}$ Endovascular therapy was implemented during the first few hours after stroke onset in SWIFT, enabling detailed analysis of the importance of time. It is essential to delineate the association of time to emergency department arrival with collateral grade and extent of CT changes, as these physiologic measures may confirm or define a therapeutic window to curb neurological injury. ${ }^{7}$ It is also 
imperative to understand how these factors mitigate the impact of recanalization therapies. Most importantly, such analyses may inform future clinicians regarding improved triage.

Our central hypothesis was that collateral grade and the resultant extent of infarction on imaging reflect the duration of ischemia, driving the chances of good outcomes following endovascular therapy. We therefore retrospectively assessed the impact of onset to door time in the SWIFT trial, correlating the time interval from last known well time to emergency department arrival with the extent of tissue injury on baseline imaging, collateral status, and final functional outcome.

\section{METHODS}

\section{Study design, participants and interventions}

The SWIFT study compared the relative performance of a novel stent-retriever device with the predicate coil retrieval device for recanalization during endovascular therapy for acute ischemic stroke. $^{6}$ The trial design, selection criteria, methodology, primary results, and detailed characteristics have been published elsewhere. ${ }^{6} 8$ Patients were randomized to mechanical thrombectomy with SOLITAIRE FR or Merci within $8 \mathrm{~h}$ of symptom onset following baseline imaging that excluded the presence of hemorrhage and confirmed occlusion in the intracranial internal carotid artery or middle cerebral artery (MCA). No penumbral or core infarct imaging criteria were used to identify potential study candidates other than absence of extensive ischemic changes. Key findings of the SWIFT trial demonstrated the superiority of the SOLITAIRE FR stent-retriever device, culminating in improved recanalization and subsequent clinical outcomes for individuals with acute ischemic stroke. ${ }^{6}$

\section{Methods and measurements}

The SWIFT trial dataset was analyzed in conjunction with measurements of baseline imaging Alberta Stroke Program Early CT Scores (ASPECTS) and angiographic collateral grades. Complete details regarding methodology of the ASPECTS scoring and angiographic collateral grade evaluation have been previously published. ${ }^{9}{ }^{10}$ In brief, evaluation of the baseline CT or MRI was conducted using the imaging archive established by the core laboratory. Two experienced readers, including a neuroradiologist and vascular neurologist with stroke imaging expertise, reviewed baseline imaging in all SWIFT studies. ASPECTS were scored using all axial slices available to reliably identify the presence of any ischemia in each topographical region of the MCA territory. Chronic changes such as leukoaraiosis, established infarcts, or atrophy were not included in generation of ASPECTS so that only acute ischemic changes could be quantified. ASPECTS were independently determined with disagreements resolved by consensus, blind to all other trial data. Collateral grade on baseline angiography was also measured using the angiography archive established by the core laboratory. The two experienced readers also reviewed baseline catheter angiography in all subjects enrolled in SWIFT, evaluating the presence of adequate information regarding collateral circulation with respect to the arterial occlusion. As collateral injections were not mandated as part of the trial protocol, variability was noted from case to case in the completeness of such information. Collateral grades prior to endovascular treatment were assessed with the American Society of Intervention and Therapeutic Neuroradiology/Society of Interventional Radiology (ASITN/SIR) scale on angiography, blind to all other data. ${ }^{11}$ Use of the ASITN/SIR grading system and scale metrics have been previously reported. ${ }^{12}$ Cases with insufficient information regarding collateral status prior to treatment were excluded from subsequent analyses. Angiographic measures of collaterals were independently determined with disagreements resolved by consensus, blind to all other trial and imaging data.

\section{Data analysis}

Statistical analyses were conducted using clinical variables obtained from the main dataset and ASPECTS scores and angiographic collateral grades were obtained as part of this post hoc study. Clinical outcomes considered were symptomatic intracranial hemorrhage by $24 \mathrm{~h}$ after treatment and functional independence at 90 days, defined as a modified Rankin Scale (mRS) of 0,1 , or 2 . The ASPECTS score was treated as an ordinal variable and the collateral score was treated as a categorical variable pooling grades 0 and 1 . Association of time to emergency department arrival with baseline ASPECTS scores, collateral grade, and subsequent outcomes was assessed using logistic regression, analyzing the impact of these individual variables with subsequent clinical outcomes defined as above. Known determinants of successful angiographic and clinical outcomes were considered as covariates, with main analyses defining the effect of time to presentation on ASPECTS score, collateral grade, reperfusion, and related clinical outcome. Baseline characteristics, including comorbid conditions, demographics, and location and severity of stroke, were compared using the Fisher exact test for categorical variables and Kruskal-Wallis test for continuous variables. A significance level of $\mathrm{p}<0.05$ was used to identify significant predictors of clinical outcomes.

\section{RESULTS}

\section{Characteristics of study subjects}

Of the entire SWIFT trial population of 144 subjects, complete data were available for 137 patients with respect to the baseline imaging and angiographic collateral grade according to the selection criteria above. Seven subjects in the trial dataset did not have anterior circulation stroke with available baseline imaging and adequate collateral injections at angiography. The mean age was $67 \pm 12$ years, with a slight predominance of women $(52 \%$ female), including pretreatment stroke severity NIH Stroke Scale (NIHSS) median score of 18 (range 8-28). The median time to emergency department arrival from last known well time was $180 \mathrm{~min}$ (IQR 95-250). The duration between last known well time and arrival was $<3 \mathrm{~h}$ in $51 \%$ and $>3 \mathrm{~h}$ in the other $49 \%$. Emergency department arrival within $3 \mathrm{~h}$ of last known well time was related to the presence of atrial fibrillation $(41.4 \%$ in those $<3 \mathrm{~h}$ vs $64.2 \%$ in those $>3 \mathrm{~h}$, $\mathrm{p}=0.010)$ and absence of prestroke disability $(17.2 \%$ vs $26.2 \%$, $\mathrm{p}=0.035)$. Early arrival was unrelated to age, sex, other vascular risk factors, baseline NIHSS score severity, initial glucose levels, or initial blood pressure.

\section{Main results}

The degree of collateral circulation noted on baseline angiography prior to endovascular therapy was strongly associated with the time duration between symptom onset and emergency department arrival (figure 1). A graded relationship was demonstrated with respect to collateral status, with mean time interval to the emergency department of $232 \pm 84 \mathrm{~min}$ in those with no or only marginal collaterals (grade 0 or $1, \mathrm{n}=32$ ), $164 \pm 99 \mathrm{~min}$ in subjects with partial collaterals (grade 2, $n=48$ ), $155 \pm 104$ min in those with slow but complete collateral filling (grade 3 , $\mathrm{n}=35)$, and $54 \pm 16 \mathrm{~min}$ in subjects with rapid and complete collaterals (grade $4, n=4$; table 1 ). Figure 2 shows the details of this relationship at the individual patient level, revealing a correlation of $-0.33(\mathrm{p}=0.0003)$. 


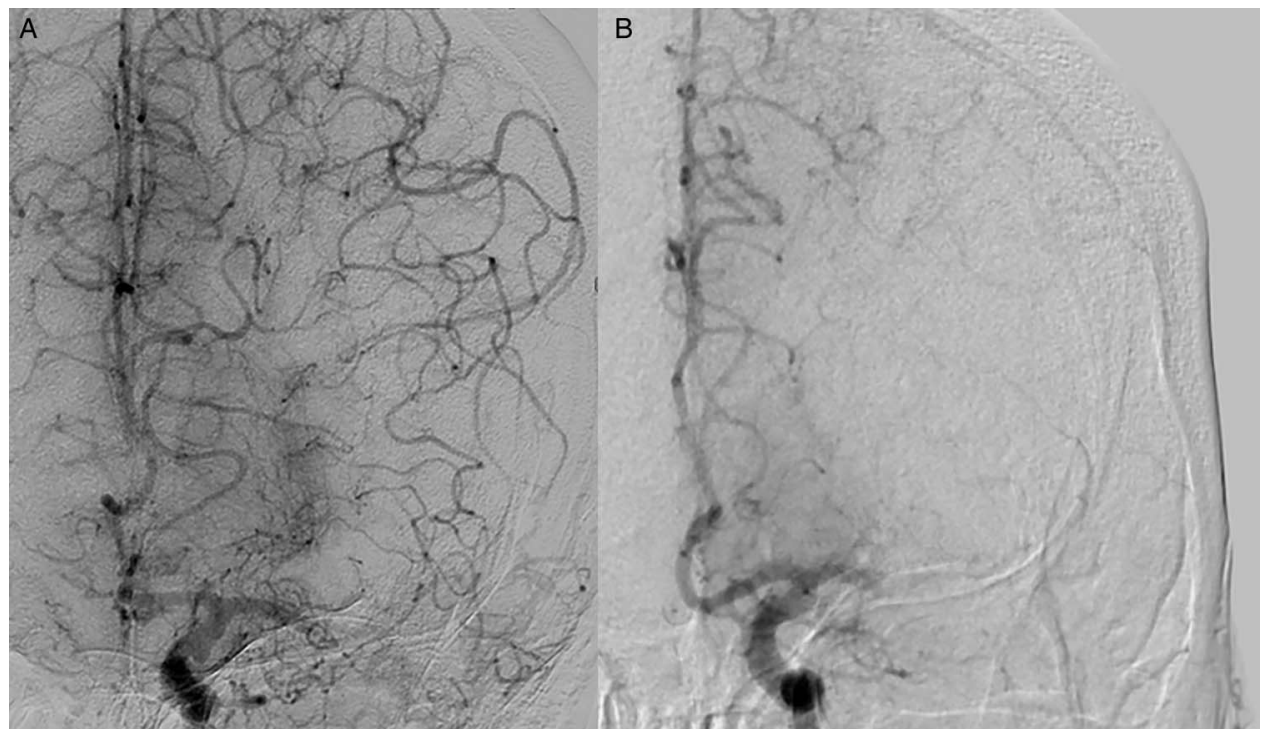

Figure 1 Case examples from two different SOLITAIRE FR With the Intention For Thrombectomy (SWIFT) subjects with acute ischemic stroke due to left middle cerebral artery occlusion. Digital subtraction angiography shows robust collateral filling in a patient arriving at the emergency department within $3 \mathrm{~h}(\mathrm{~A})$ and another case where only marginal collaterals are demonstrated with presentation to hospital beyond $5 \mathrm{~h}$ (B).

More extensive ischemic injury on baseline imaging was noted in subjects presenting at longer intervals from symptom onset to emergency department arrival (figure 3). Later presentation was associated with more extensive ischemic injury at baseline (median ASPECTS 8 (IQR 7-9) in those $>3 \mathrm{~h}$ vs 9 (IQR $8-10$ ) in those within $3 \mathrm{~h}, \mathrm{p}=0.015$ ). Furthermore, multivariable analyses identified presentation as an independent predictor of extensive baseline ischemic changes (ASPECTS $\leq 7$ : OR $1.008 / \mathrm{min}$ (95\% CI 1.003 to 1.014$), \mathrm{p}=0.003$ ).

Interestingly, the time interval between symptom onset and emergency department arrival was unrelated to the likelihood of successful angiographic reperfusion (Thrombolysis In Myocardial Infarction (TIMI) grade 2 or $3: 53.7 \%$ vs $54.1 \%, p=1.00$; TIMI grade $2 \mathrm{~b}$ or $3: 67.2 \%$ vs $60.7 \%, \mathrm{p}=0.47)$. Earlier presentation to the emergency department, however, demonstrated a strong relationship with better 90-day mRS clinical outcomes (mRS 0-2: $50.0 \%$ vs $22.2 \%, \mathrm{p}=0.0015$ ).

\section{DISCUSSION}

The time between the last known well time in acute ischemic stroke and emergency department arrival was strongly associated with the degree of collateral circulation and extent of early ischemic changes on baseline imaging. Shorter time intervals before hospital arrival were linked with better collaterals and smaller infarcts. Interestingly, this time interval demonstrated no relationship with respect to the likelihood of successful recanalization with endovascular therapy, yet the clinical outcomes of this population were strongly related to this pre-hospital time interval. These findings suggest that individual or patientspecific factors associated with the underlying pathophysiology may be important determinants of outcome after endovascular therapy for acute ischemic stroke. Indeed, prior studies have shown that collateral circulation and stroke severity are influential in determining the response to reperfusion and, decisively, the long-term clinical outcomes or disability following ischemic stroke. ${ }^{1} 10$ Our analysis of SWIFT establishes an important and practical link between such detailed imaging variables and the time course of these stroke patients before reaching the emergency department.
The relationship between patient-specific factors and healthcare delivery metrics in stroke care is important as it provides opportunities to improve pre-hospital and emergency medicine triage of such patients. Symptom onset occurs when collateral circulation is insufficient to sustain perfusion of the ischemic territory in the brain. ${ }^{13}$ The time course of collateral failure has been difficult to delineate across a large population of stroke patients, yet our data suggest that earlier arrival at the emergency department may catch patients before subsequent collateral demise. As collaterals fail, infarct evolution may ensue with more extensive early ischemic changes noted on baseline imaging. There is undoubtedly a complex relationship between collateral status, duration of ischemia, and infarct core. The biological determinants or mechanisms and the causal relationships that link collateral failure with infarct extension over time deserve further investigation. These findings suggest that shortening time intervals to hospital arrival and beyond may maximize ideal candidates for endovascular therapy, stroke patients with more robust collaterals and smaller infarct cores. Several groups have already implemented quality improvement initiatives to shorten all time intervals in stroke care delivery, including strategies to streamline triage prior to endovascular therapy. $^{14-16}$

The impact of shortening the time from symptom onset to emergency department arrival remains to be proven in a prospective trial that accounts for patient-specific factors such as imaging. The PHANTOM-S mobile CT study includes imaging obtained in the field via specialized ambulances, yet the marked heterogeneity of collateral perfusion or variability across patients may make it difficult to prove that such an approach is costeffective in a large population. ${ }^{17}$ It is therefore important to demonstrate the association between time intervals in healthcare delivery and individual patient pathophysiology.

The recent positive results of MR CLEAN and preliminary reports of similar positive findings in other randomized trials of second-generation embolectomy therapies make more urgent the need to understand determinants of benefit from endovascular therapy. Although trials may demonstrate superiority of a treatment strategy, patient-specific factors are undoubtedly pivotal in the course of individual stroke outcomes. 
Table 1 Relationship between collateral grades and baseline variables and outcomes

\begin{tabular}{|c|c|c|c|c|c|}
\hline Subject characteristics & Collateral grade $0-1(n=32)$ & Collateral grade $2(n=48)$ & Collateral grade $3(n=35)$ & Collateral grade $4(n=4)$ & $p$ Value \\
\hline \multicolumn{6}{|l|}{ Age } \\
\hline Mean \pm SD (N) & $67.3 \pm 11.3(32)$ & $66.8 \pm 11.5(48)$ & $67.8 \pm 13.1(35)$ & $71.5 \pm 14.5(4)$ & 0.5231 \\
\hline [Median] (min, max) & {$[66.0](46.0,85.0)$} & {$[69.5](42.0,88.0)$} & {$[70.0](31.0,85.0)$} & {$[74.0](53.0,85.0)$} & \\
\hline Female gender & $31.3 \%(10 / 32)$ & $64.6 \%(31 / 48)$ & $51.4 \%(18 / 35)$ & $50.0 \%(2 / 4)$ & 0.3242 \\
\hline \multicolumn{6}{|l|}{ Body mass index } \\
\hline Mean \pm SD (N) & $30.3 \pm 6.9(24)$ & $29.3 \pm 7.4(40)$ & $30.4 \pm 8.1(29)$ & $26.6 \pm 3.6(3)$ & 0.9578 \\
\hline [Median] (min, max) & {$[30.0](20.1,47.2)$} & {$[28.9](16.0,60.8)$} & {$[27.1](17.8,52.6)$} & {$[27.0](22.8,30.0)$} & \\
\hline Pre-stroke mRS 0 & $63.0 \%(17 / 27)$ & $63.4 \%(26 / 41)$ & $85.7 \%(24 / 28)$ & $75.0 \%(3 / 4)$ & 0.0429 \\
\hline Intravenous tPA delivered and failed & $28.1 \%(9 / 32)$ & $50.0 \%(24 / 48)$ & $51.4 \%(18 / 35)$ & $66.7 \%(2 / 3)$ & 0.0647 \\
\hline \multicolumn{6}{|l|}{ Baseline risk factors } \\
\hline Myocardial disease & $28.1 \%(9 / 32)$ & $37.5 \%(18 / 48)$ & $37.1 \%(13 / 35)$ & $0.0 \%(0 / 4)$ & 0.8108 \\
\hline Systemic hypertension & $62.5 \%(20 / 32)$ & $58.3 \%(28 / 48)$ & $77.1 \%(27 / 35)$ & $75.0 \%(3 / 4)$ & 0.2589 \\
\hline Diabetes mellitus & $34.4 \%(11 / 32)$ & $25.0 \%(12 / 48)$ & $17.1 \%(6 / 35)$ & $75.0 \%(3 / 4)$ & 0.5209 \\
\hline Hyperlipidemia & $43.8 \%(14 / 32)$ & $45.8 \%(22 / 48)$ & $62.9 \%(22 / 35)$ & $75.0 \%(3 / 4)$ & 0.0383 \\
\hline Current or previous smoker & $43.8 \%(14 / 32)$ & $47.9 \%(23 / 48)$ & $31.4 \%(11 / 35)$ & $0.0 \%(0 / 4)$ & 0.0415 \\
\hline Atrial fibrillation & $56.3 \%(18 / 32)$ & $43.8 \%(21 / 48)$ & $54.3 \%(19 / 35)$ & $25.0 \%(1 / 4)$ & 0.3540 \\
\hline Previous ischemic stroke & $9.4 \%(3 / 32)$ & $25.0 \%(12 / 48)$ & $2.9 \%(1 / 35)$ & $0.0 \%(0 / 4)$ & 0.3265 \\
\hline \multicolumn{6}{|l|}{ NIHSS pre-procedure } \\
\hline Mean $\pm S D(N)$ & $18.9 \pm 4.9(32)$ & $17.0 \pm 4.8(48)$ & $17.3 \pm 4.3(35)$ & $15.5 \pm 4.5(4)$ & 0.0664 \\
\hline [Median] (min, max) & {$[19.0](8.0,27.0)$} & {$[17.0](8.0,28.0)$} & {$[18.0](8.0,26.0)$} & {$[17.0](9.0,19.0)$} & \\
\hline \multicolumn{6}{|l|}{ Glucose at baseline } \\
\hline Mean $\pm S D(N)$ & $153.7 \pm 65.2(32)$ & $139.8 \pm 74.8(47)$ & $124.1 \pm 33.4(35)$ & $99.0 \pm 10.4(4)$ & 0.0134 \\
\hline [Median] (min, max) & {$[137.5](86.0,354.0)$} & {$[121.0](87.0,587.0)$} & {$[113.0](84.0,239.0)$} & {$[102.5](84.0,107.0)$} & \\
\hline \multicolumn{6}{|l|}{ SBP at baseline } \\
\hline Mean \pm SD (N) & $148.8 \pm 24.5(32)$ & $136.7 \pm 23.1(48)$ & $137.0 \pm 23.5(35)$ & $137.8 \pm 9.9(4)$ & 0.0384 \\
\hline [Median] (min, max) & {$[146.0](108.0,196.0)$} & {$[135.0](98.0,182.0)$} & {$[135.0](72.0,194.0)$} & {$[138.0](126.0,149.0)$} & \\
\hline \multicolumn{6}{|l|}{ DBP at baseline } \\
\hline Mean \pm SD (N) & $80.7 \pm 14.2(32)$ & $75.8 \pm 19.4(48)$ & $74.8 \pm 14.4(35)$ & $72.3 \pm 13.3(4)$ & 0.1279 \\
\hline [Median] (min, max) & {$[80.5](51.0,110.0)$} & {$[72.0](32.0,130.0)$} & {$[73.0](45.0,103.0)$} & {$[77.0](53.0,82.0)$} & \\
\hline \multicolumn{6}{|l|}{ ASPECTS at baseline } \\
\hline Mean $\pm S D(N)$ & $7.8 \pm 1.8(32)$ & $8.8 \pm 1.1(48)$ & $8.9 \pm 1.2(35)$ & $9.0 \pm 0.8(4)$ & 0.0002 \\
\hline [Median] (min, max) & {$[8.0](3.0,10.0)$} & {$[9.0](5.0,10.0)$} & {$[9.0](7.0,10.0)$} & {$[9.0](8.0,10.0)$} & \\
\hline \multicolumn{6}{|l|}{ ASPECTS at $24 \mathrm{~h}$} \\
\hline Mean $\pm S D(N)$ & $1.4 \pm 1.6(32)$ & $5.9 \pm 2.7(48)$ & $7.5 \pm 2.4(34)$ & $6.8 \pm 1.9(4)$ & 0.0000 \\
\hline [Median] (min, max) & {$[0.5](0.0,5.0)$} & {$[6.0](0.0,10.0)$} & {$[8.0](0.0,10.0)$} & {$[7.5](4.0,8.0)$} & \\
\hline \multicolumn{6}{|l|}{ Time to presentation ( $\mathrm{min}$ ) } \\
\hline Mean \pm SD (N) & $232.0 \pm 84.0(31)$ & $164.1 \pm 98.7(48)$ & $155.2 \pm 104.0(33)$ & $53.7 \pm 16.1$ (3) & 0.0003 \\
\hline [Median] (min, max) & {$[230.0](24.0,382.0)$} & {$[160.0](0.0,440.0)$} & {$[135.0](23.0,385.0)$} & {$[47.0](42.0,72.0)$} & \\
\hline \multicolumn{6}{|l|}{ Time to imaging (min) } \\
\hline Mean \pm SD (N) & $306.7 \pm 76.3(31)$ & $284.0 \pm 78.4(47)$ & $279.4 \pm 89.0(33)$ & $208.0 \pm 69.4(4)$ & 0.0263 \\
\hline [Median] (min, max) & {$[301.0](144.0,431.0)$} & {$[260.0](134.0,472.0)$} & {$[265.0](123.0,465.0)$} & {$[185.5](155.0,306.0)$} & \\
\hline \multicolumn{6}{|l|}{ Time to treatment $(\mathrm{min})$} \\
\hline Mean \pm SD (N) & $332.8 \pm 83.5(27)$ & $305.8 \pm 80.0(41)$ & $291.5 \pm 85.6$ (34) & $217.0 \pm 69.6(4)$ & 0.0070 \\
\hline [Median] (min, max) & {$[326.0](154.0,459.0)$} & {$[283.0](169.0,478.0)$} & {$[287.0](137.0,476.0)$} & [194.0] $(165.0,315.0)$ & \\
\hline \multicolumn{6}{|c|}{ Time to recanalization or end of procedure (min) } \\
\hline Mean $\pm S D(N)$ & $62.7 \pm 41.1(26)$ & $55.0 \pm 41.3(41)$ & $41.9 \pm 27.2(33)$ & $65.0 \pm 41.9(4)$ & 0.1126 \\
\hline [Median] (min, max) & {$[54.0](8.0,178.0)$} & {$[43.0](7.0,196.0)$} & {$[36.0](5.0,135.0)$} & {$[62.5](28.0,107.0)$} & \\
\hline Intracranial hemorrhage & $34.4 \%(11 / 32)$ & $35.4 \%(17 / 48)$ & $17.1 \%(6 / 35)$ & $0.0 \%(0 / 4)$ & 0.1379 \\
\hline \multicolumn{6}{|l|}{ NIHSS at day 7 or discharge } \\
\hline Mean \pm SD (N) & $23.3 \pm 12.0(27)$ & $15.0 \pm 11.1(47)$ & $7.8 \pm 10.2(35)$ & $5.8 \pm 5.3(4)$ & 0.0000 \\
\hline [Median] (min, max) & {$[20.0](6.0,42.0)$} & {$[15.0](1.0,42.0)$} & {$[4.0](0.0,42.0)$} & {$[5.5](0.0,12.0)$} & \\
\hline Good clinical outcome (mRS 0-2) & $10.0 \%(3 / 30)$ & $26.8 \%(11 / 41)$ & $56.3 \%(18 / 32)$ & $66.7 \%(2 / 3)$ & 0.0001 \\
\hline TIMI success (2 or 3 ) & $37.9 \%(11 / 29)$ & $52.3 \%(23 / 44)$ & $68.6 \%(24 / 35)$ & $25.0 \%(1 / 4)$ & 0.1033 \\
\hline $\mathrm{TICl}$ success ( $2 \mathrm{~b}$ or 3 ) & $41.4 \%(12 / 29)$ & $65.9 \%(29 / 44)$ & $77.1 \%(27 / 35)$ & $50.0 \%(2 / 4)$ & 0.0187 \\
\hline \multicolumn{6}{|l|}{$\mathrm{mRS}$ at day 90} \\
\hline mRS 0 & $0.0 \%(0 / 30)$ & $4.9 \%(2 / 41)$ & $25.0 \%(8 / 32)$ & $33.3 \%(1 / 3)$ & 0.0000 \\
\hline mRS 1 & $3.3 \%(1 / 30)$ & $7.3 \%(3 / 41)$ & $21.9 \%(7 / 32)$ & $33.3 \%(1 / 3)$ & \\
\hline $\mathrm{mRS} 2$ & $6.7 \%(2 / 30)$ & $14.6 \%(6 / 41)$ & $9.4 \%(3 / 32)$ & $0.0 \%(0 / 3)$ & \\
\hline $\mathrm{mRS} 3$ & $6.7 \%(2 / 30)$ & $24.4 \%(10 / 41)$ & $12.5 \%(4 / 32)$ & $0.0 \%(0 / 3)$ & \\
\hline
\end{tabular}


Table 1 Continued

\begin{tabular}{|c|c|c|c|c|c|}
\hline Subject characteristics & Collateral grade $0-1 \quad(n=32)$ & Collateral grade $2(n=48)$ & Collateral grade $3(n=35)$ & Collateral grade $4(n=4)$ & p Value \\
\hline mRS 4 & $23.3 \%(7 / 30)$ & $19.5 \%(8 / 41)$ & $21.9 \%(7 / 32)$ & $33.3 \%(1 / 3)$ & \\
\hline mRS 5 & $3.3 \%(1 / 30)$ & $4.9 \%(2 / 41)$ & $0.0 \%(0 / 32)$ & $0.0 \%(0 / 3)$ & \\
\hline $\mathrm{mRS} 6$ & $56.7 \%(17 / 30)$ & $24.4 \%(10 / 41)$ & $9.4 \%(3 / 32)$ & $0.0 \%(0 / 3)$ & \\
\hline \multicolumn{6}{|l|}{ TIMI score after device } \\
\hline TIMI 0 & $51.7 \%(15 / 29)$ & $34.1 \%(15 / 44)$ & $25.7 \%(9 / 35)$ & $75.0 \%(3 / 4)$ & 0.0771 \\
\hline TIMI 1 & $10.3 \%(3 / 29)$ & $13.6 \%(6 / 44)$ & $5.7 \%(2 / 35)$ & $0.0 \%(0 / 4)$ & \\
\hline TIMI 2 & $17.2 \%(5 / 29)$ & $13.6 \%(6 / 44)$ & $14.3 \%(5 / 35)$ & $0.0 \%(0 / 4)$ & \\
\hline TIMI 3 & $20.7 \%(6 / 29)$ & $38.6 \%(17 / 44)$ & $54.3 \%(19 / 35)$ & $25.0 \%(1 / 4)$ & \\
\hline \multicolumn{6}{|l|}{$\mathrm{TICl}$ score after device } \\
\hline $\mathrm{TICl} 0$ & $34.5 \%(10 / 29)$ & $13.6 \%(6 / 44)$ & $14.3 \%(5 / 35)$ & $50.0 \%(2 / 4)$ & 0.0534 \\
\hline $\mathrm{TICl} 1$ & $10.3 \%(3 / 29)$ & $13.6 \%(6 / 44)$ & $5.7 \%(2 / 35)$ & $0.0 \%(0 / 4)$ & \\
\hline $\mathrm{TICl} 2 \mathrm{a}$ & $13.8 \%(4 / 29)$ & $6.8 \%(3 / 44)$ & $2.9 \%(1 / 35)$ & $0.0 \%(0 / 4)$ & \\
\hline $\mathrm{TICl} 2 \mathrm{~b}$ & $20.7 \%(6 / 29)$ & $25.0 \%(11 / 44)$ & $22.9 \%(8 / 35)$ & $25.0 \%(1 / 4)$ & \\
\hline $\mathrm{TICl} 3$ & $20.7 \%(6 / 29)$ & $40.9 \%(18 / 44)$ & $54.3 \%(19 / 35)$ & $25.0 \%(1 / 4)$ & \\
\hline
\end{tabular}

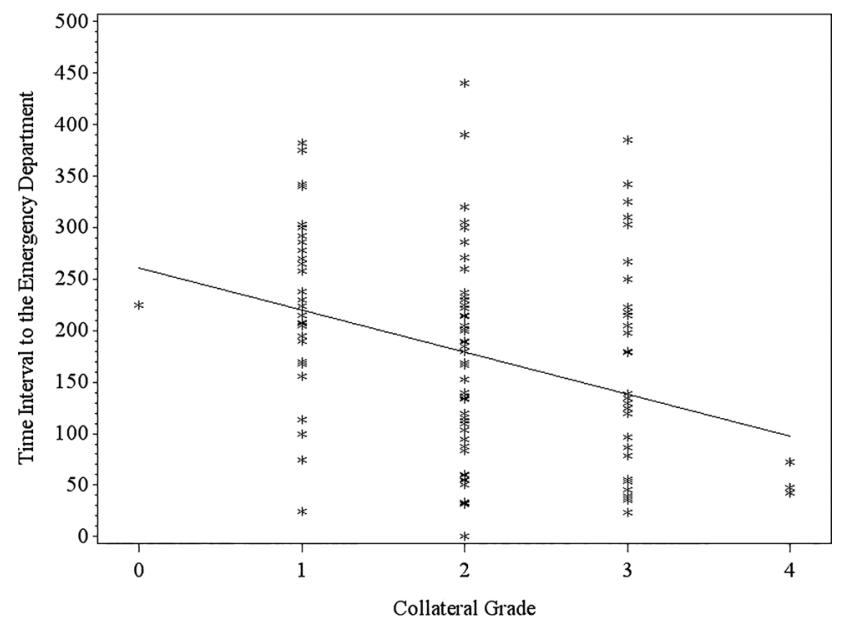

Figure 2 Plot of collateral grade with respect to time interval to arriving at the emergency department.
Generalizability of endovascular stroke therapy including prehospital triage, emergency department evaluation, and imaging-guided approaches in routine clinical practice is imperative. What works best in the community or on a local basis at various times of the day or night remains a critical question. Each randomized trial design embeds pivotal details regarding the overall treatment strategy from onset of symptoms forward, evaluating far more than the impact of a particular device or thrombectomy procedure. It is important to ascertain the impact of timing on collateral grade, evolving infarction, and long-term outcomes. This is a mandatory step before concluding that the endovascular therapy works for an entire population rather than in a specific individual where the timing, physiology, and many other factors are just right.

Future systems of stroke care may enhance the utility of endovascular therapy if it is understood how timing in an individual patient may improve their outcome. The advanced triage and rapid evaluation metrics embedded in ESCAPE and SWIFT PRIME may further augment the recent progress of MR CLEAN. ${ }^{14}{ }^{18}$ Decreasing the time interval from symptom onset
Figure 3 Case examples from SOLITAIRE FR With the Intention For Thrombectomy (SWIFT) subjects demonstrating the differences in early ischemic changes on CT associated with left middle cerebral artery occlusion. CT reveals subtle hypodensity restricted to the left caudate region in a patient presenting to hospital within $3 \mathrm{~h}(\mathrm{~A})$ and another case where extensive hypodensity is seen throughout the left middle cerebral artery territory with presentation to the emergency department $4 \mathrm{~h}$ after symptom onset (B).

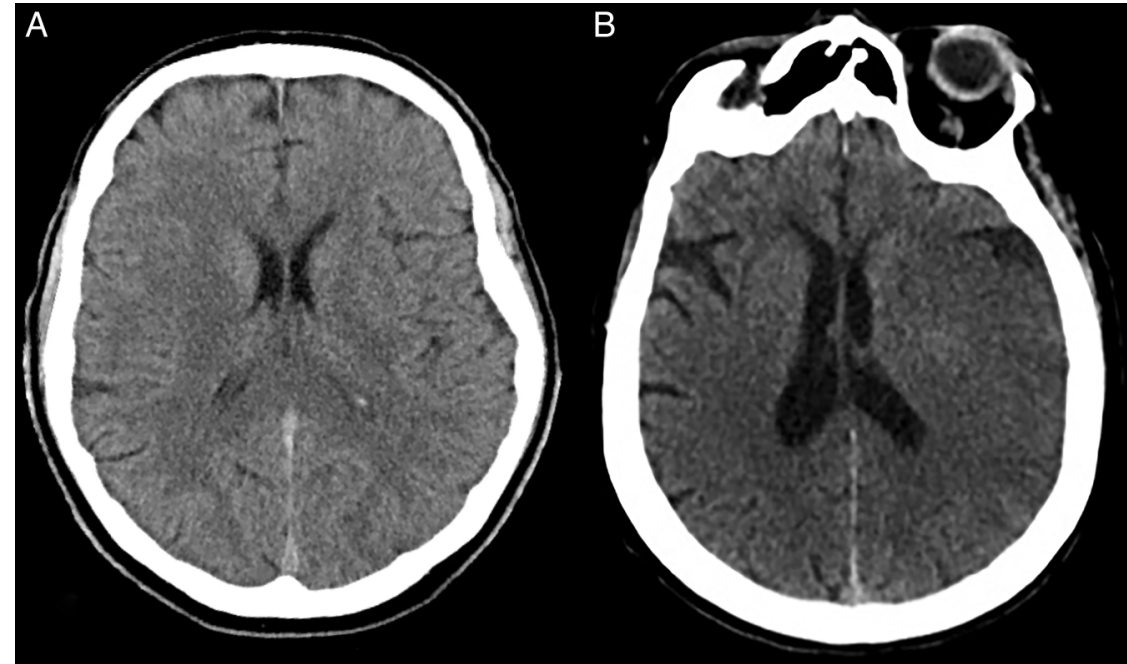


to emergency department arrival, a pivotal variable in SWIFT, may be a formative step.

Limitations of these findings include the fact that this is a post hoc study, so the findings should be considered exploratory rather than confirmatory. The findings are based on readings by expert imaging reviewers from an experienced core laboratory; local clinicians may not replicate these results at the time of patient evaluation and decision-making. Furthermore, there are limitations associated with the nature of the ASPECTS and ASITN/SIR scoring systems. For example, both scoring systems are characterized by relatively unimodal scale characteristics where the majority of patients cluster around an ASPECTS score of 7 or, alternatively, an ASITN/SIR grade of 2 . Importantly, it should be noted that ASPECTS determination of infarction based on CT measures during the earliest hours after symptom onset might be fraught with error. Although we explored many potential determinants of the time interval between symptom onset and emergency department arrival, unmeasured factors could have contributed as well. Finally, associations observed at the group level regarding the impact of time may not be accurate in any individual case.

\section{CONCLUSIONS}

Time was a critical factor in the successful clinical outcomes of patients with stroke treated with endovascular therapy in SWIFT. Shorter times from symptom onset to emergency department arrival were associated with better collaterals, smaller established infarcts, and better clinical outcome after revascularization.

Contributors DSL, RJ, RGN, TGJ and JLS conceived the study, designed the trial, obtained research funding, and supervised the conduct of the trial and data collection. RJ and JLS undertook recruitment of participating centers and patients and managed the data, including quality control. DSL, RJ, RGN, TGJ, HLL and JLS provided statistical advice on study design and analyzed the data. DSL drafted the manuscript and all authors contributed substantially to its revision. DSL takes responsibility for the paper as a whole.

Competing interests None declared.

Ethics approval The study was approved by the local institutional review board or ethics committee with concomitant registration of the clinical trial.

Provenance and peer review Not commissioned; externally peer reviewed.

\section{REFERENCES}

1 Liebeskind DS, Tomsick TA, Foster LD, et al. Collaterals at angiography and outcomes in the interventional management of stroke (IMS) III trial. Stroke 2014;45:759-64.

2 Kidwell CS, Jahan R, Gornbein J, et al. A trial of imaging selection and endovascular treatment for ischemic stroke. N Eng J Med 2013;368:914-23.

3 Broderick JP, Palesch YY, Demchuk AM, et al. Endovascular therapy after intravenous t-PA versus t-PA alone for stroke. N Eng J Med 2013;368:893-903.

4 Ciccone A, Valvassori L, Nichelatti M, et al. Endovascular treatment for acute ischemic stroke. N Eng J Med 2013;368:904-13.

5 Berkhemer OA, Fransen PS, Beumer D, et al. A randomized trial of intraarterial treatment for acute ischemic stroke. N Engl J Med 2015:372:11-20.

6 Saver JL, Jahan R, Levy El, et al. Solitaire flow restoration device versus the Merci Retriever in patients with acute ischaemic stroke (SWIFT): a randomised, parallel-group, non-inferiority trial. Lancet 2012;380:1241-9.

7 Hill MD, Rowley HA, Adler F, et al. Selection of acute ischemic stroke patients for intra-arterial thrombolysis with pro-urokinase by using ASPECTS. Stroke 2003;34:1925-31.

8 Saver JL, Jahan R, Levy El, et al. Solitaire with the intention for thrombectomy (SWIFT) trial: design of a randomized, controlled, multicenter study comparing the Solitaire flow restoration device and the Merci Retriever in acute ischaemic stroke. Int J Stroke 2014;9:658-68.

9 Liebeskind DS, Jahan R, Nogueira RG, et al. Serial Alberta Stroke Program Early CT Score from baseline to 24 hours in Solitaire Flow Restoration with the Intention for Thrombectomy study: a novel surrogate end point for revascularization in acute stroke. Stroke 2014;45:723-7.

10 Liebeskind DS, Jahan R, Nogueira RG, et al. Impact of collaterals on successful revascularization in Solitaire FR with the intention for thrombectomy. Stroke 2014;45:2036-40.

11 Higashida RT, Furlan AJ, Roberts $\mathrm{H}$, et al. Trial design and reporting standards for intra-arterial cerebral thrombolysis for acute ischemic stroke. Stroke 2003;34:e109-137.

12 Zaidat 00, Yoo AJ, Khatri P, et al. Recommendations on angiographic revascularization grading standards for acute ischemic stroke: a consensus statement. Stroke 2013:44:2650-63.

13 Liebeskind DS. Collateral circulation. Stroke 2003;34:2279-84.

14 Demchuk AM, Goyal M, Menon BK, et al. Endovascular treatment for Small Core and Anterior circulation Proximal occlusion with Emphasis on minimizing $\mathrm{CT}$ to recanalization times (ESCAPE) trial: methodology. Int I Stroke 2015;10:429-38.

15 Goyal M, Menon BK, Hill MD, et al. Consistently achieving computed tomography to endovascular recanalization $<90$ minutes: solutions and innovations. Stroke 2014:45:e252-256.

16 Menon BK, Almekhlafi MA, Pereira VM, et al. Optimal workflow and process-based performance measures for endovascular therapy in acute ischemic stroke: analysis of the Solitaire FR thrombectomy for acute revascularization study. Stroke 2014;45:2024-9.

17 Weber JE, Ebinger M, Rozanski M, et al. Prehospital thrombolysis in acute stroke: results of the PHANTOM-S pilot study. Neurology 2013;80:163-8.

18 ClinicalTrials.gov. Solitaire With the Intention For Thrombectomy as PRIMary Endovascular Treatment (SWIFT PRIME) trial. https://clinicaltrials.gov/ct2/show/ NCT01657461 (accessed 15 Jan 2015). 